\title{
Perfil demográfico de idosos da cidade de Joinville, Santa Catarina: Estudo de base domiciliar
}

\section{Demographic profile of the elderly in the city of Joinville, Santa Catarina: a household survey}

\section{Marco Fabio Mastroeni ${ }^{1}$ \\ Gilmar Sidnei Erzinger ${ }^{2}$ \\ Silmara Salete de Barros Silva Mastroeni ${ }^{3}$ \\ Nilza Nunes da Silva ${ }^{4}$ \\ Maria de Fátima Nunes Marucci ${ }^{5}$ \\ 'Departamento de Biologia, Universidade da Região de Joinville \\ ${ }^{2}$ Departamento de Farmácia, Universidade da Região de Joinville \\ ${ }^{3}$ Curso de Nutrição da Associação Educacional Luterana Bom Jesus/IELUSC \\ ${ }^{4}$ Departamento de Epidemiologia, Faculdade de Saúde Pública, Universidade de São Paulo \\ ${ }^{5}$ Departamento de Nutrição, Faculdade de Saúde Pública, Universidade de São Paulo}

Fonte de financiamento: Universidade da Região de Joinville-UNIVILLE. Processo $n^{\circ}$ 26/02 de 28/ 02/2002.

Correspondência: Marco Fabio Mastroeni. Departamento de Biologia, Universidade da Região de Joinville - UNIVILLE - Campus Universitário S/N, Bairro Bom Retiro - Joinville, SC CEP: 89.223-251. Email: mmastroeni@univille.br

\section{Resumo}

Objetivo: Identificar o perfil demográfico dos idosos residentes na área urbana de Joinville-SC. Metodologia: Utilizando-se amostragem por conglomerados em múltiplos estágios nos setores censitários urbanos de Joinville, 660 idosos foram entrevistados em suas casas por meio de questionários. A análise estatística para variáveis categóricas consistiu em testes de associação segundo o sexo, utilizando-se o Teste do Qui-Quadrado. Em todas as análises foi considerado o nível de significância $p<0,05$. Resultados: A média de idade dos idosos foi de 69,8 anos, sendo a maioria $(57,6 \%)$ mulheres. Grande parte $(62,0 \%)$ dos idosos vivia com o cônjuge, e $33,0 \%$ eram viúvos (as); o grupo etário predominante foi o de 60-69 anos (54,8\%); o grupo étnico com maior representatividade foi o de cor branca (89,5\%); a maioria $(84,5 \%)$ era natural de Santa Catarina, sendo o Rio Grande do Sul o Estado que apresentou maior representatividade de imigrantes (3,9\%). Em relação à escolaridade, $72,9 \%$ não completaram o ensino fundamental, 9,2\% eram analfabetos e apenas 5,3\% ingressaram no ensino médio. A maior parte $(45,6 \%)$ dos idosos recebia até dois salários mínimos, e 50,3\% das mulheres eram dependentes financeiramente. Em relação ao IMC, 32,8\% apresentaram valores médios acima do adequado. Conclusão: O perfil demográfico do grupo estudado mostrou características similares a de outros estudos realizados com idosos no país. Por se tratar de um grupo etário em rápido crescimento no mundo, as características demográficas são imprescindíveis para o estabelecimento de políticas públicas de saúde que visem controlar o estado de saúde da população.

Palavras-chave: Epidemiologia. Envelhecimento. Idosos. Joinville. 


\section{Abstract}

Objective: To identify the demographic profile of the elderly people living in the urban area of Joinville-SC. Method: using multistage cluster sampling of Joinville urban sectors, 660 elderly individuals were interviewed in their homes with the use of questionnaires. Chi-square test was used for association tests for categorical variables according to gender. A level of significance of $p<0.05$ was considered for all analyses. The differences between genders were considered statistically significant when $p<0.05$. Results: The average age was 69.8 years, and most (57.6\%) of the elderly were women. The majority (62.0\%) was living with a partner, and $33.0 \%$ were widows/ ers; the predominant age group was 60-69 years (54.8\%); the most highly represented ethnical group was the Caucasian (89.5\%); most individuals $(84.5 \%)$ were born in Santa Catarina, and Rio Grande do Sul was the original state of most immigrants (3.9\%). As to schooling, $72.9 \%$ did not complete elementary school, $9.2 \%$ were illiterate, and only $5.3 \%$ had gone to high school. Concerning income, $45.6 \%$ received up to two Brazilian minimum wages, and $50.3 \%$ of the women were financially dependent. In relation to $\mathrm{BMI}, 32.8 \%$ presented mean values above appropriate. Conclusion: The demographic profile of this population was similar to other studies with the elderly in the country. Given it is an age group that is growing increasing faster in the country and in the world, having information on their demographic characteristics is necessary to establish public health policies aimed at controlling and promoting this population's health.

Keywords: Epidemiology. Aging. Elderly. Joinville.

\section{Introdução}

Já na década de 80 , Ramos et al. ${ }^{1}$ alertavam para o rápido processo de envelhecimento da população brasileira, particularmente sobre suas implicações sociais e em termos de saúde pública. Nessa mesma época, Kalache et al. ${ }^{2}$ estimaram que, no período de 1980 a 2000, paralelamente a um crescimento da população total de 56\%, haveria aumento da população idosa no Brasil de mais de $100 \%$. O grupo etário com 60 anos de idade e acima passaria de $5 \%$ da população total, em 1960 , para $14,0 \%$ em 2025. Segundo o último Censo realizado no Brasil, em 2000, a população de pessoas com 60 anos de idade e acima no Brasil representava $8,5 \%$ da população total, registrando aumento de $100 \%$ em 20 anos $^{3}$. Nos próximos 20 anos, a população idosa do Brasil poderá ultrapassar a 30 milhões de pessoas e deverá representar cerca de $13 \%$ da população ao final deste período 4 .

Durante o século XX, ocorreram importantes mudanças no tipo de doenças e nas causas de morte da população brasileira. Nas primeiras quatro décadas do século $\mathrm{XX}$, o Brasil apresentou características demográfico-sanitárias encontradas em países tipicamente em desenvolvimento, tendo como maior causa de mortalidade as doenças infecto-contagiosas. A partir de 1940, começou a ocorrer incremento das doenças crônicas não transmissíveis, as quais passaram a representar a causa primária da mortalidade da população até os dias de hoje ${ }^{5}$. Segundo Carvalho e Garcia ${ }^{6}$, a partir de 1940, e até os anos 60, a população brasileira manteve-se quase estável, com distribuição etária praticamente constante. Nesse período, houve significante declínio da mortalidade e leve queda da fecundidade.

Em grande parte, essa mudança epidemiológica deveu-se a uma estabilização na estrutura etária, que apresentou baixas oscilações nas taxas de natalidade e mortalidade até meados do século $\mathrm{XX}^{7}$. Este quadro mudou entre as décadas de 40 e 70 , 
quando o coeficiente de mortalidade populacional passou a apresentar declínio acentuado devido à diminuição na mortalidade infantil. A partir da década de 70, a expectativa de vida da população brasileira passou a aumentar gradativamente ao longo dos anos, ocorrendo o inverso com a taxa de fecundidade, que vem mantendo declínio até os dias de hoje ${ }^{5,8}$. Se, a partir do ano 2000, não houver mais declínio da fecundidade no Brasil, o que é de todo improvável, mesmo assim continuará o processo de envelhecimento da população, até que desapareçam totalmente de sua configuração etária os efeitos diretos e indiretos da alta fecundidade do passado ${ }^{6}$.

Acompanhando o processo de envelhecimento da população mundial, a população idosa de Joinville também vem aumentando consideravelmente ao longo dos anos. Nos últimos 20 anos, houve considerável aumento de $151 \%$ na população idosa de Joinville, passando de 11.263 idosos em 1980, para 28.236 idosos em $2000^{3}$. Este expressivo crescimento do número de idosos na cidade de Joinvile realça a necessidade de se obter dados demográficos da população idosa de forma a se propor políticas públicas no sentido de direcionar o envelhecimento saudável da população. Este estudo tem como objetivo descrever as características sociodemográficas de idosos residentes na cidade de Joinville$\mathrm{SC}$, a partir de inquérito domiciliar. Com base nesses dados será possível traçar metas e organizar programas de saúde que visem a melhoria da qualidade de vida da população investigada.

\section{Metodologia}

Os dados apresentados fazem parte de um estudo maior, de base populacional, transversal, realizado mediante inquérito domiciliar e que avaliou o estado nutricional e o consumo de macronutrientes de idosos da cidade de Joinville-SC. A população de estudo foi composta por 28.236 idosos ${ }^{9}$ com idade igual ou superior a 60 anos, de ambos os sexos, não institucio- nalizados e residentes na área urbana da cidade de Joinville-SC, no ano de 2002.

\section{Composição da amostra}

O tamanho da amostra foi definido a partir dos recursos financeiros, recursos humanos e de tempo, disponíveis para o desenvolvimento do estudo. Assim, determinou-se como aceitável entrevistar entre 600 e 800 idosos. Nessas circunstâncias, o erro de amostragem para estimar proporções em processo aleatório simples manteve-se abaixo de $5 \%$, indicando um número mínimo de 384 entrevistas para coeficiente de confiança igual a 95\%, conforme Silva ${ }^{10}$. O número mínimo, fixado entre 600 e 800 idosos, atendeu à necessidade de proteger à precisão fixada devido ao efeito de homogeneidade intra setor censitário $^{11}$, tomado como unidade primária de amostragem.

O sorteio de 800 idosos foi realizado em dois estágios, sob o método de partilha proporcional ao tamanho - $\mathrm{PPT}^{10,11}$, tomando-se o setor censitário e a moradia como respectivas unidades de primeiro e segundo estágio. Todos os idosos localizados em cada moradia foram entrevistados.

Adotando-se 20 entrevistas como sendo o número máximo tolerado a ser obtido dentro de cada unidade primária ${ }^{11}$, estimou-se o número de moradias a serem visitadas, em cada setor, através da razão: $20 \times(2,1 / 10)=95,24$, onde a razão 2,1/10 é o número de idosos residentes (28.236 idosos/132.388 domicílios) que era esperado encontrar por moradia 9 .

Calculou-se o número total de moradias a serem sorteadas a partir do número máximo de 800 idosos, estipulado para compor a amostra, seguindo-se a razão: $800 /(2,1 / 10)=3.809,5$, arredondado para 4.000 moradias. Dividiu-se o número total de moradias a serem sorteadas pelo número de moradias a serem visitadas em cada setor, fornecendo, como resultado, o número de setores censitários que compõem a amostra de primeiro estágio: 4.000/95,24 $=41,99$. Finalmente, a amostra de idosos 
foi obtida multiplicando-se o número máximo de entrevistas em cada setor pelo número de setores que compõem a amostra de primeiro estágio: $20 \times 42=840$.

\section{Processo de sorteio}

A partir dos 385 setores censitários urbanos dos distritos de Joinville e Pirabeiraba, que representaram o município de Joinville, foram mantidos apenas 382 setores, classificados como "comum ou não especial” e em "situação urbana”, segundo a Fundação IBGE 4 . Destes, foram sorteados 42 setores censitários, mediante sorteio sistemático com probabilidade proporcional ao número de domicílios ${ }^{11}$.

Para o sorteio de moradias no segundo estágio, procedeu-se à contagem de moradias por quadra de cada setor, e a seguir foram sorteadas 100 moradias. Em cada quadra, todos os domicílios sorteados foram visitados, identificando-se aqueles nos quais residiam idosos e solicitando-lhes que participassem do estudo. A fração global de amostragem $(f)$ foi calculada pela expressão $f=\left((42 \times D j) / \Sigma_{D j}\right) \times(100 / D j)$, onde $D j$ é o número de moradias existentes em cada setor, contadas pelo censo demográfico do ano 2000.

\section{Coleta dos dados}

Na primeira etapa do estudo, que consistiu da coleta de dados em domicílio mediante entrevista, 660 idosos foram entrevistados, representando 78,5\% da amostra probabilística. Nesta fase, foram coletados os seguintes dados: nome, logradouro, nacionalidade/naturalidade, estado civil, cor ou grupo étnico, escolaridade e renda. Na segunda etapa do estudo, realizada nos postos de saúde, foram coletados os dados antropométricos, bioquímicos e dietéticos, comparecendo 221 idosos, aproximadamente $33,5 \%$ da amostra.

Para este trabalho foram considerados apenas os dados de identificação, socioeconômicos e de antropometria (peso e estatura) como uma primeira abordagem:
- Identificação, contendo informações sobre o nome, endereço, nacionalidade, naturalidade, estado civil, data de nascimento, cor ou grupo étnico relatado e sexo;

- Dados socioeconômicos, contendo questões sobre escolaridade e renda;

- Antropometria, a partir das medidas de peso e estatura determinou-se o Índice de Massa Corporal (IMC). Tais medidas foram efetuadas da seguinte forma:

\section{Peso}

Os idosos foram pesados em balança digital da marca Soehnle, com capacidade de 150 kg e divisão de 100 g. No momento da mensuração, o idoso estava vestindo o mínimo de roupas possível (com os bolsos vazios, sem casaco, chapéu, boné, cinto, xale e demais acessórios que acarretassem aumento do peso), sem calçados, e posicionado no centro da balança, de forma a distribuir o peso do corpo entre os pés, conforme técnica de Gordon e col. $(1988)^{12}$.

\section{Estatura}

A estatura foi mensurada utilizando-se estadiômetro portátil da marca Seca, com capacidade para até $200 \mathrm{~cm}$, de acordo com a técnica de Gordon e col. (1988) ${ }^{12}$.

Como critério de análise para o IMC, adotou-se padrão de referência de Kuczmarski e col. (2000) ${ }^{13}$, que descreve como sendo adequados os valores de IMC compreendidos entre $22,4-27,1 \mathrm{~kg} / \mathrm{m}^{2}$ para homens, e 21,7-28,4 kg/m² para mulheres.

\section{Aspecto ético}

A identificação dos participantes foi sigilosa, e os dados armazenados com o pesquisador responsável pelo estudo. Quando houve concordância de participação, os voluntários assinaram o Termo de Consentimento Informado, elaborado de acordo com as normas da Resolução n ${ }^{\circ}$ 196/ 1996, do Conselho Nacional de Saúde, sendo fornecida uma cópia a cada participante do estudo. Quaisquer dúvidas surgidas 
ao longo do estudo foram esclarecidas pelo pesquisador responsável. Para o desenvolvimento do estudo, o projeto foi aprovado pelos Comitês de Ética em Pesquisa da Faculdade de Saúde Pública da USP e da Universidade da Região de Joinville.

\section{Análise estatística}

Os dados foram analisados utilizandose o módulo "survey", cujos procedimentos foram precedidos do código " $s v y$ " do software STATA 7.0 ${ }^{14}$, que incluiu efeito do plano de amostragem por conglomerados sobre as estimativas e respectivos intervalos de confiança.

A análise estatística para variáveis categóricas (estado civil, grupo etário, cor, escolaridade, renda e IMC), consistiu em testes de associação, segundo o sexo, utilizando-se o Teste do Qui-Quadrado. Os valores estimados para níveis de significância foram comparados ao valor padrão de 0,05 para o nível de significância.

\section{Resultados}

Foram registrados 665 idosos, sendo entrevistados 660 efetivamente. Cinco idosos foram excluídos do estudo por não terem sido localizados ou por se recusarem a participar da pesquisa.

A maioria (57,6\%) dos idosos pertencia ao sexo feminino. As idades mínima e máxima encontradas foram 60 e 95 anos, respectivamente, sendo a média de idade de 69,8 anos. Mais da metade $(54,8 \%)$ apresentou idade abaixo de 70 anos e, apenas $11,9 \%$, idade acima de 80 anos, não havendo diferença significativa para grupo etário entre os sexos (Tabela 1).

Em relação à naturalidade, 84,5\% eram catarinenses. Dos indivíduos não nascidos em Santa Catarina, o Estado que apresentou maior representatividade de imigrantes foi o Rio Grande do Sul (3,9\%), seguido do Paraná (3,3\%), São Paulo $(2,1 \%)$ e demais Estados $(5,1 \%)$. Uma pequena parcela $(1,1 \%)$ declarou ser estrangeira, predominantemente de origem européia.
Em relação ao estado civil, a maioria (62,0\%) dos idosos vivia com o cônjuge (casados ou em união consensual). Um terço (33\%) era constituído por viúvos(as) e poucos eram solteiros $(1,8 \%)$ ou separados $(3,2 \%)$. As mulheres viúvas foram quatro vezes mais $(48,4 \%)$ freqüentes do que os homens $(12,1 \%)$, e a maioria $(83,6 \%)$ dos idosos do sexo masculino relatou viver com o cônjuge. Houve diferença estatisticamente significante entre os idosos quanto ao estado civil (Tabela 1).

A maioria $(89,5 \%)$ dos idosos relatou pertencer à cor branca. A cor parda foi a segunda mais relatada: $10,4 \%$ e $8,2 \%$, respectivamente, para os sexos masculino e feminino, não havendo diferença estatisticamente significante para cor ou grupo étnico entre os sexos (Tabela 1).

Um total de 481 idosos (72,9\%) declarou não ter concluído o ensino fundamental, $1^{\text {a }}$ a $8^{\mathrm{a}}$ séries, e apenas $5,3 \%$ haviam concluído pelo menos o ensino médio. Excetuando-se as categorias de não alfabetizados e ensino superior, as mulheres relataram maior freqüência nas demais categorias. Houve diferença estatisticamente significante entre os sexos para a escolaridade (Tabela 1).

Quanto à renda, a grande maioria (78,5\%) dos idosos era dependente financeiramente ou recebia até dois salários mínimos (R\$ 400,00). Apenas 2,7\% recebiam cinco ou mais salários mínimos. As mulheres relataram ser dependentes financeiramente em maior (50,3\%) freqüência do que os homens $(9,3 \%)$, havendo diferença estatisticamente significante entre os sexos. O contrário ocorreu para os que recebiam até três salários mínimos, sendo os idosos do sexo masculino os mais freqüentes (72,1\%) (Tabela 1).

Os dados de peso, estatura e IMC encontram-se descritos na Tabela 2. Homens e mulheres não apresentaram diferença em relação ao peso, mas os homens apresentaram maior média de estatura $(166,3 \mathrm{~cm})$ em relação às mulheres (155,1 cm), havendo diferença estatisticamente significante $(\mathrm{p}<0,05)$ entre os sexos. A média do IMC dos 
Tabela 1 - Características sociodemográficas dos idosos da área urbana de Joinville, segundo sexo.

Table 1 - Socio-demographic characteristics of the elderly of the urban area of Joinville by gender.

\begin{tabular}{|c|c|c|c|c|c|c|c|}
\hline \multirow[t]{3}{*}{ Dados demográficos } & \multicolumn{4}{|c|}{ Sexo } & & & \multirow{3}{*}{$p$} \\
\hline & \multicolumn{2}{|c|}{ Masculino } & \multicolumn{2}{|c|}{ Feminino } & \multicolumn{2}{|c|}{ Total } & \\
\hline & $\mathrm{n}$ & $\%$ & $\mathrm{n}$ & $\%$ & $\mathrm{n}$ & $\%$ & \\
\hline \multicolumn{8}{|l|}{ Estado civil } \\
\hline Solteiro & 5 & 1,8 & 7 & 1,8 & 12 & 1,8 & 0,0000 \\
\hline Casado e União consensual & 234 & 83,6 & 175 & 46,1 & 409 & 62,0 & \\
\hline Desquitado e Separado & 7 & 2,5 & 14 & 3,7 & 21 & 3,2 & \\
\hline Viúvo & 34 & 12,1 & 184 & 48,4 & 218 & 33,0 & \\
\hline \multicolumn{8}{|l|}{ Grupo etário (anos) } \\
\hline $60-69$ & 158 & 56,5 & 204 & 53,8 & 362 & 54,8 & 0,4535 \\
\hline $70-79$ & 93 & 33,2 & 127 & 33,4 & 220 & 33,3 & \\
\hline $80+$ & 29 & 10,3 & 49 & 12,8 & 78 & 11,9 & \\
\hline \multicolumn{8}{|l|}{ Cor ou grupo étnico } \\
\hline Branca & 244 & 87,1 & 347 & 91,3 & 591 & 89,5 & 0,1110 \\
\hline Negra & 6 & 2,1 & 2 & 0,5 & 8 & 1,2 & \\
\hline Parda & 29 & 10,4 & 31 & 8,2 & 60 & 9,1 & \\
\hline Amarela e Indígena & 1 & 0,4 & 0 & 0 & 1 & 0,2 & \\
\hline \multicolumn{8}{|l|}{ Escolaridade } \\
\hline Não alfabetizado & 13 & 4,6 & 48 & 12,6 & 61 & 9,2 & 0,0001 \\
\hline Alfabetizado & 30 & 10,7 & 22 & 5,8 & 52 & 7,9 & \\
\hline Ensino fundamental incompleto & 201 & 71,7 & 280 & 73,7 & 481 & 72,9 & \\
\hline Ensino fundamental completo & 15 & 5,4 & 16 & 4,2 & 31 & 4,7 & \\
\hline Ensino médio e superior, completos e incompletos & 21 & 7,5 & 14 & 3,7 & 35 & 5,3 & \\
\hline \multicolumn{8}{|l|}{ Renda (SM = R\$ 200,00) } \\
\hline Dependente & 26 & 9,3 & 191 & 50,3 & 217 & 32,9 & 0,0000 \\
\hline Até 2 SM & 145 & 51,8 & 156 & 41,0 & 301 & 45,6 & \\
\hline 2-3 SM & 57 & 20,3 & 23 & 6,1 & 80 & 12,1 & \\
\hline 3-5 SM & 38 & 13,6 & 6 & 1,6 & 44 & 6,7 & \\
\hline $5+$ & 14 & 5,0 & 4 & 1,0 & 18 & 2,7 & \\
\hline Total & 280 & 100 & 380 & 100 & 660 & 100 & \\
\hline
\end{tabular}

voluntários examinados foi $26,8 \mathrm{~kg} / \mathrm{m}^{2}$, onde as mulheres apresentaram média de IMC significativamente $(\mathrm{p}<0,05)$ superior $\left(28,1 \mathrm{~kg} / \mathrm{m}^{2}\right)$ a dos homens $\left(25,2 \mathrm{~kg} / \mathrm{m}^{2}\right)$.

Quando separados em relação aos percentis de referência para IMC, segundo Kuczmarski e col. (2000) ${ }^{13}$, apesar da maioria dos homens e das mulheres ter apresentado valores médios de IMC normais, uma grande parcela $(32,8 \%)$ dos idosos apresentou valores médios de IMC elevados (Tabela 3), havendo diferença estatisticamente significante $(\mathrm{p}<0,05)$ dos percentis de IMC entre os sexos. Nota-se que a diferença ocorreu para percentis de IMC inadequado (Tabela 3).

\section{Discussão}

Estudos de base domiciliar estão sujeitos a fatores inesperados do ambiente que, eventualmente, podem interferir na coleta dos dados. Neste estudo, um fator importante que prejudicou a coleta de dados em domicílio foram as eleições de 2002, que ocorreram na mesma época em que a pesquisa foi desenvolvida: setembro a novembro de 2002. Por várias vezes, os idosos se recusaram a atender os entrevistadores, acreditando ser uma abordagem política, mesmo estando os entrevistadores devidamente identificados com o crachá das universidades envolvidas no estudo. Outro fa- 
Tabela 2 - Peso, estatura e IMC de 217 idosos da área urbana de Joinville-SC, segundo sexo. Table 2 - Weight, height and BMI of 217 elderly of the urban area of Joinville-SC, by gender.

\begin{tabular}{llcccc}
\hline \multirow{2}{*}{$\begin{array}{l}\text { Variáveis } \\
\text { antropométricas }\end{array}$} & \multicolumn{4}{c}{ Sexo } \\
& & $\mathrm{n}=87$ & $\mathrm{n}=130$ & $\mathrm{n}=217$ & \\
\hline Peso $(\mathrm{kg})$ & Média & 69,8 & 67,8 & 68,7 & 0,2980 \\
& Erro padrão & 1,62 & 1,44 & 1,24 & \\
& I.C. 95\% & {$[66,9 ; 73,5]$} & {$[64,3 ; 70,1]$} & {$[66,2 ; 71,2]$} & \\
\hline Estatura $(\mathrm{cm})^{*}$ & Média & 166,3 & 155,1 & 160,0 & 0,0000 \\
& Erro padrão & 1,08 & 0,78 & 0,94 & \\
& I.C. 95\% & {$[164,1 ; 168,4]$} & {$[153,5 ; 156,7]$} & {$[158,1 ; 161,9]$} & \\
\hline IMC $\left(\mathrm{kg} / \mathrm{m}^{2}\right)^{*}$ & Média & 25,2 & 28,1 & 26,8 & 0,0000 \\
& Erro padrão & 0,41 & 0,55 & 0,41 & \\
& I.C. 95\% & {$[24,4 ; 26,0]$} & {$[26,9 ; 29,2]$} & {$[25,9 ; 27,7]$} & \\
\hline
\end{tabular}

${ }^{*} \mathrm{p}<0,05$.

Tabela 3 - Freqüências relativas (\%) dos percentis IMC de 217 idosos da área urbana de Joinville-SC, por sexo, segundo proposto por Kuczmarski e col. (2000).

Table 3 - Relative frequencies (\%) of BMI percentile of 217 elderly of the urban area of Joinville-SC, by gender, as proposed by Kuczmarski et al. (2000).

\begin{tabular}{lccccc}
\hline & \multicolumn{3}{c}{ Sexo } & & \\
IMC & Masculino & Feminino & Total & $\chi^{2}$ & $p$ \\
& $(\%)$ & $(\%)$ & $(\%)$ & & \\
\hline Inadequado (insuficiente): valores <P25 & 16,0 & 5,2 & 10,0 & 3,82 & 0,0270 \\
Adequado: valores entre P25 e P75 & 60,0 & 55,0 & 57,2 & & \\
Inadequado (elevado): valores >P75 & 24,0 & 39,8 & 32,8 & & \\
Total & 100,0 & 100,0 & 100,0 & & \\
\hline
\end{tabular}

tor que também prejudicou a coleta dos dados foi a indefinição dos idosos em relatar a cor ou grupo étnico a que pertenciam. Apesar de ser a mesma definição utilizada pelo IBGE no último censo ${ }^{15}$ para a obtenção de dados sobre etnia, no momento de relatar a qual grupo o idoso pertencia, pareceu haver certo receio por parte de alguns voluntários em afirmar pertencer à raça negra. Tais características devem ser levadas em conta em estudos do tipo inquérito.

Assim como em outros estudos desenvolvidos no Brasil ${ }^{16-23}$, neste estudo também foi verificada maior freqüência de idosos do sexo feminino. Estudos desenvolvidos em outros países também descreveram as mulheres como mais freqüentes na população ${ }^{24,25}$. A predominância do sexo feminino confirma a maior expectativa de vida deste grupo etário ${ }^{20}$. Esta característica tem sido atribuída a: menor exposição a determinados fatores de risco, notadamente no trabalho; menor prevalência de tabagismo e consumo de bebida alcoólica; diferenças quanto à atitude em relação a doenças e incapacidades; e, por último, maior cobertura da assistência ginecoobstétrica $^{26}$. Segundo a Fundação $\mathrm{IBGE}^{4}$, a característica da mulher residir na cidade também pode beneficiar na maior expectativa de vida, especialmente as viúvas, devido à proximidade com seus filhos, dos serviços especializados de saúde e de outros facilitadores do cotidiano. 
O padrão atual, com predomínio de mulheres acima de 60 anos em proporções que aumentam conforme progride a idade, é um aspecto de importância no planejamento dos programas locais de assistência à terceira idade. Inquéritos populacionais têm mostrado que as mulheres na velhice apresentam mais problemas de saúde e sentem-se menos saudáveis do que os homens, situação que é agravada pelos maiores índices de viuvez e solidão, e pelo prolongamento do tempo de vida média ${ }^{27}$.

A maior freqüência de mulheres viúvas encontrada neste estudo, cerca de quatro vezes maior que a dos homens, coincide com os resultados descritos por outros autores $^{21,22}$. Lebrão ${ }^{22}$, em estudo conduzido no Município de São Paulo no ano de 2000, também relatou a freqüência quatro vezes maior de mulheres viúvas. Uma pesquisa realizada com idosos no Rio Grande do Sul mostrou que, além das mulheres apresentarem maior freqüência de viuvez, estas também predominaram na área urbana das cidades onde o estudo foi desenvolvido $^{17}$. Outro estudo realizado em Fortaleza-CE, também revelou maior freqüência de mulheres viúvas ${ }^{26}$.

Segundo a OPAS ${ }^{28}$, as mulheres e os homens envelhecem de maneira diferente. No que se refere à esperança de vida, a vantagem da mulher, em parte, é biológica. Longe de ser o sexo frágil, o sexo feminino parece ter maior capacidade de recuperação que o masculino, em todas as idades. É possível, também, que a vantagem biológica das mulheres possa estar associada ao fato de, na vida adulta, possuírem hormônios que as protegem contra doenças do coração. Schlenker ${ }^{29}$ coloca que o fato de as mulheres superarem os homens em relação à expectativa de vida pode estar relacionado a fatores genéticos ou ambientais, ou uma combinação de ambos. Lebrão ${ }^{22}$ destaca o estado civil como sendo a característica demográfica mais expressiva da população idosa, com diferenciais por sexo, resultado, principalmente, da maior expectativa de vida da mulher.

Os dados da distribuição demográfica da população analisada mostraram o predomínio de uma população homogênea, sendo a maioria catarinense. Embora esta característica esteja sendo modificada ao longo dos anos, Joinville ainda é uma cidade que sofre pouca influência migratória dos outros Estados do Brasil. A característica de uma população homogênea do Estado e, conseqüentemente, de Joinville, também pode ser observada quando se investiga a cor ou o grupo étnico relatado, onde poucos $(10,5 \%)$ relataram não ser de cor branca. Essa baixa influência migratória dos Estados vizinhos e o padrão étnico homogêneo observado sugerem que Joinville ainda mantém as características estabelecidas pela colonização européia. Esta é uma característica importante, pois reflete o estilo de vida da população. Por ainda manter hábitos antigos da colonização européia, como, por exemplo, deslocar-se a pé ou de bicicleta e cultivar flores e hortaliças no quintal de casa, a população idosa ainda preserva um estilo de vida saudável.

Outra característica que também contribuiu para manter o estilo de vida saudável desta população foi sua baixa média de idade, 69,8 anos, mostrando que a população estudada foi constituída predominantemente por idosos “jovens” (60-69 anos), tendo como aspecto positivo o fato de grande parte $(62,0 \%)$ ainda viver com o cônjuge, característica esta importante para o aumento da qualidade de vida do idoso. A característica de idoso "jovem" é facilmente verificada quando se analisa por grupo etário, onde a maioria $(54,8 \%)$ dos idosos relatou idade inferior a 70 anos. Apenas $11,9 \%$ dos idosos relataram possuir 80 ou mais anos de idade. Este perfil também foi verificado em outros estudos ${ }^{16-18,20,23,26}$.

O perfil do idoso descrito neste estudo, com baixa renda e escolaridade, coincide com o retratado por outros autores ${ }^{1,16,20,21,26}$. A baixa escolaridade relatada, onde a maioria $(72,9 \%)$ dos que freqüentaram a escola revelou não ter completado o ensino fundamental, também era esperada. É importante colocar que, neste estudo, os dados de renda (individual) e escolaridade foram 
coletados como categóricos, sem discriminar anos de estudo e valor exato da renda. Nas décadas de 10 a 40 do século XX, períodos nos quais foi constituída a maioria da população pesquisada, havia menor freqüência à escola, priorizando-se o trabalho em detrimento da educação. Nessa época, grande parte da população estudava até a quarta série do primário, visto que não havia exigência de maior escolaridade para se conseguir melhores cargos e, conseqüentemente, melhor renda. Desta forma, embora não seja possível afirmar exatamente quantos anos de estudo os indivíduos desta pesquisa cursaram, é pertinente supor que a maioria também tenha concluído apenas a primeira parte do ensino fundamental ( $1^{\text {a }}$ a $4^{\text {a }}$ série). Acredita-se que esta característica será substancialmente modificada para as próximas gerações, devido à maior possibilidade de freqüência à escola e, também, à grande competitividade do mercado de trabalho atual.

Quanto à renda, o fato de as mulheres terem relatado maior $(50,3 \%)$ dependência financeira em relação aos homens $(9,3 \%)$ vem ao encontro do que foi comentado anteriormente, considerando que as mulheres nascidas até o começo do século passado desenvolviam atividades mais relacionadas ao lar e ao cuidado dos filhos do que os homens, responsáveis pela busca do sustento familiar. Neste estudo, os dados de renda coletados referem-se aos indivíduos entrevistados, não à renda familiar. Segundo a Fundação $\mathrm{IBGE}^{4}$, quando o assunto é escolaridade e renda, as diferenças entre homens e mulheres são expressivas. Mesmo que ambos tenham a mesma média de anos de estudo, os homens ainda ganham mais do que as mulheres. Essa desigualdade de rendimentos se mantém em todos os Estados e regiões, e em todas as classes de anos de estudo. Segundo a Fundação IBGE ${ }^{4}$, tanto as mulheres com grau de escolaridade igual ou inferior a três anos de estudo quanto aquelas com grau de escolaridade superior a 11 anos de estudo ganham menos do que os homens com o mesmo grau de escolaridade.
É possível que a baixa renda individual relatada pela maioria da população investigada esteja relacionada à escolaridade, visto que $34,2 \%$ dos idosos (dados não mostrados) que recebiam renda de até dois salários mínimos afirmaram não ter completado o ensino fundamental. Atualmente, os idosos economicamente ativos acabam sendo pressionados pelo sistema competitivo do mercado de trabalho, principalmente no que diz respeito ao baixo grau de escolaridade que a maioria apresenta, o que dificulta a busca por melhores salários. Este quadro deve ser alterado para as próximas gerações, principalmente pelo fato de a mulher estar buscando cada vez mais seu espaço no mercado de trabalho, necessitando, conseqüentemente, de melhor nível educacional.

O aumento do índice de alfabetização entre os idosos é um fato altamente positivo para esse grupo populacional. O maior acesso às informações divulgadas pelos meios de comunicação mantém o indivíduo ativo e participante, promovendo a busca de melhores empregos e renda, facilitando a utilização dos serviços de saúde e a receptividade aos programas educacionais e sanitários. Essas características tornam a alfabetização um dos indicadores mais precisos para se verificar o nível socioeconômico de uma população ${ }^{27}$.

Outra variável de fundamental importância para conhecer o perfil sociodemográfico de uma população diz respeito ao IMC. Neste estudo, optou-se por utilizar os padrões de IMC estabelecidos por Kuczmarski e col. (2000) ${ }^{13}$, os valores compreendidos como sendo normais entre 22,4 e $27,1 \mathrm{~kg} / \mathrm{m}^{2}$ para homens, e 21,7 e 28,4 $\mathrm{kg} / \mathrm{m}^{2}$ para mulheres. Adotando-se esse padrão, a maioria $(57,2 \%)$ dos indivíduos desse estudo foi classificada como sendo adequada quanto ao IMC (entre P25 e P75). Mesmo assim, a considerável prevalência de ambos os sexos (24,0\% homens e $39,8 \%$ mulheres) com IMC elevado (valores >P75), revelou um quadro preocupante. Quando a classificação da WHO (1997) ${ }^{30}$ foi utilizada para indivíduos com sobrepeso, 
descrito como IMC igual ou superior a 25,0 $\mathrm{kg} / \mathrm{m}^{2}$, a maior prevalência de obesidade também foi encontrada no sexo feminino, dados que são consistentes com os retratados por outros estudos realizados no Brasil $^{19,20,31-34}$ e em outros países ${ }^{24,35-37}$.

Segundo Marucci e Barbosa (2003) ${ }^{34}$, em estudo realizado no município de São Paulo, no ano de 2000 (Projeto SABE), $21,7 \%$ dos homens e $40,5 \%$ das mulheres apresentaram sobrepeso e obesidade. Outro estudo ${ }^{38}$ também realizado em São Paulo, com 154 mulheres atendidas no Ambulatório de Saúde da Mulher Climatérica USP, revelou que apenas $24,0 \%$ das mulheres apresentaram-se adequadas em relação ao estado nutricional, e 75,3\% apresentaram IMC igual ou superior a $25,0 \mathrm{~kg} / \mathrm{m}^{2}$.

$\mathrm{O}$ excesso de peso é um dos principais fatores de risco de uma série de doenças crônicas não transmissíveis, entre elas, a hipercolesterolemia, o diabetes, as doenças cardiovasculares, algumas formas de câncer, entre outras, e representa, atualmente, um preocupante desafio à saúde pública.

O sobrepeso é um problema eminentemente urbano, com algumas diferenciações por região. Na região sul, o excesso de peso é também elevado na área rural. Tais resultados podem estar refletindo a presença diferenciada de fatores determinantes de sobrepeso relacionados a condições de vida, história ocupacional e de estilos de vida, principalmente atividade física e consumo alimentar, conforme gênero, nas diversas realidades sociais brasileiras ${ }^{19}$.

Não menos importante que os indivíduos apresentando IMC elevado são os indivíduos que apresentaram IMC classificado como insuficiente $(\mathrm{P}<25)$, valores $<22,4 \mathrm{~kg} / \mathrm{m}^{2}$ para homens, e $<21,7 \mathrm{~kg} / \mathrm{m}^{2}$ para mulheres, segundo Kuczmarski e col. $(2000)^{13}$. Neste estudo, $10,0 \%$ dos idosos apresentaram IMC insuficiente, sendo nos homens cerca de três vezes mais $(16,0 \%)$ freqüentes que nas mulheres $(5,2 \%)$. Marucci e Barbosa (2003) $)^{34}$ encontraram resultado similar no município de São Paulo, onde o sexo masculino também apresentou maior prevalência de baixo peso.
Barreto e col. (2003) ${ }^{33}$, também relataram maior freqüência de baixo peso no sexo masculino em estudo desenvolvido na cidade de Bambuí, MG.

A literatura científica sobre peso corporal tem enfatizado os riscos associados a sobrepeso, mostrando pouca atenção aos problemas relacionados a baixo peso. Uma questão importante abordada por vários estudos sobre obesidade, e que merece atenção, diz respeito ao direcionamento da informação: excesso de peso é sempre danoso à saúde, sendo melhor o menor peso $^{39}$. O IMC insuficiente também traz conseqüências sérias e, em algumas situações, irreversíveis a qualquer pessoa, merecendo ser mais bem investigado. A detecção precoce de eventual alteração do estado nutricional de uma população é essencial para o desenvolvimento de trabalhos preventivos ou de intervenção terapêutica, evitando o desenvolvimento de doenças no futuro.

O desafio maior do século XXI será cuidar de uma população de mais de 32 milhões de idosos, a maioria com níveis socioeconômico e educacional baixos e uma alta prevalência de doenças crônicas não transmissíveis ${ }^{40}$. O processo de envelhecimento tem um impacto significativo em inúmeros fatores que afetam o desenvolvimento das sociedades e o relativo bem-estar, não só das pessoas idosas, mas também das populações jovens.

Por se tratar de um grupo etário em rápido crescimento no mundo, as características sociodemográficas são imprescindíveis para o estabelecimento de políticas públicas de saúde que visem promover a saúde da população e controlar as doenças.

\section{Agradecimentos}

À Fundação IBGE-Joinville, pelo prestativo auxílio no planejamento da coleta de dados; a UNIVILLE, pela estrutura física e de recursos humanos, e a Coordenação de Aperfeiçoamento de Pessoal de Nível Superior-CAPES, pela bolsa de estudo concedida. 


\section{Referências}

1. Ramos LR, Veras RP, Kalache A. Envelhecimento populacional: uma realidade brasileira. Rev Saúde Pública 1987; 21 (3): 211-24.

2. Kalache A, Veras RP, Ramos LR. O envelhecimento da população mundial: um desafio novo. Rev Saúde Pública 1987; 21 (3): 200-10.

3. Fundação IBGE. Censo demográfico 2000: Resultados do universo. IBGE - Instituto Brasileiro de Geografia e Estatística. 2003: [3]. Disponível em URL: http:// www.ibge.gov.br/home/ estatistica/populacao/ censo2000/tabelabrasil111.shtm [Acessado em 31 de julho de 2003.

4. Fundação IBGE. Censo demográfico 2000: Agregados de setores censitários dos resultados do universo. Documentação dos arquivos de dados. Rio de Janeiro; julho: 2002.

5. Cruz IBM, Alho CS. Envelhecimento populacional: panorama epidemiológico e de saúde do Brasil e do Rio Grande do Sul. In: Jeckel Neto E, Cruz IBM (Orgs.). Aspectos biológicos e geriátricos do envelhecimento II. Porto Alegre: EDIPUCRS; 2000. p. 175-91.

6. Carvalho JAM, Garcia RA. O envelhecimento da população brasileira: um enfoque demográfico. Cad Saúde Pública 2003; 19 (3): 725-33.

7. Camargo ABM, Saad PM. A transição demográfica no Brasil e seu impacto na estrutura etária da população. In: Fundação SEADE. O idoso na grande São Paulo. São Paulo: SEADE; 1990.

8. Fundação IBGE. Tábua de vida. Evolução da Mortalidade - 2001, Brasil. IBGE - Instituto Brasileiro de Geografia e Estatística. 2001: [7]. Disponível em URL: http:// www.ibge.gov. br/home/estatistica/populacao/ tabuadevida/evolucao_da_mortalidade_2001.shtm [Acessado em 10 de agosto de 2003]

9. Fundação IBGE. Censo demográfico 2000: Cartograma e folhas para fins estatísticos. Descrição dos Setores Censitários. Mapa dos Setores Censitários. Joinville-SC. [CD ROM]. Rio de Janeiro; 2000a.

10. Silva NN. Amostragem probabilística: Um curso introdutório. $2^{\mathrm{a}}$ ed. rev. São Paulo: EDUSP; 2000.

11. Kish L. Survey Sampling. New York: John Wiley \& Sons; 1965.

12. Gordon CC, Chumlea WC, Roche AF. Stature, recumbent length, and weight. In: Lohman TG, Roche AF, Martorell R. Anthropometric Standardization Reference Manual. Champaign, Illinois: Human Kinetics Books; 1988. p. 3-8.

13. Kuczmarski MF, Kuczmarski RJ, Najjar M. Descriptive anthropometric reference data for older Americans. JAm Diet Assoc 2000; 100 (1): 59-66.

14. StataCorp. Stata Statistical Software: Release 7.0. College Station (TX): Stata Corporation; 2001
15. Fundação IBGE. Manual do Recenseador CD 1.09: Censo 2000. Rio de Janeiro. 2000b.

16. Ramos LR, Rosa TEC, Oliveira ZM, Medina MCG, Santos FRG. Perfil do idoso em área metropolitana na região sudeste do Brasil: resultados de inquérito domiciliar. Rev Saúde Pública 1993; 27 (2): 87-94.

17. Rio Grande do Sul. Os idosos do Rio Grande do Sul: estudo multidimensional de suas condições de vida: relatório de pesquisa. Conselho Estadual do Idoso. Porto Alegre: CEI; 1997.

18. Ramos LR, Toniolo JN, Cendoroglo MS, Garcia JT, Najas MS, Perracini M et al. Two-year follow-up study of elderly residents in S. Paulo, Brazil: methodology and preliminary results. Rev Saúde Pública 1998; 32 (5): 397-407.

19. Tavares EL, Anjos LA. Perfil antropométrico da população idosa brasileira. Resultados da Pesquisa Nacional sobre Saúde e Nutrição. Cad Saúde Pública 1999; 15(4): 327-33.

20. Cabrera MAS, Jacob Filho W. Obesidade em idosos: prevalência, distribuição e associação com hábitos e comorbidades. Arq Bras Endocrinol Metab 2001; 45 (5): 494501.

21. Lima Costa MFFL, Uchoa E, Guerra HL, Firmo JOA, Vidigal PG, Barreto SM. The Bambuí health and ageing study (BHAS): methodological approach and preliminary results of a population-based cohort study of the elderly in Brazil. Rev Saúde Pública 2000; 38 (2): 126-35.

22. Lebrão ML. O projeto SABE em São Paulo: uma visão panorâmica. In: Lebrão ML, Duarte YAO (Orgs.). SABE: Saúde, Bem-estar e Envelhecimento. O Projeto SABE no Município de São Paulo: uma abordagem inicial. Brasília: Organização Pan-Americana de Saúde; 2003. p. 33-43.

23. Pereira RS, Curioni CC, Veras R. Perfil demográfico da população idosa no Brasil e no Rio de Janeiro. Textos Envelhecimento: 2003; 6(1).

24. Vellas BJ, Hunt WC, Romero LJ, Koehler KM, Baumgartner RN, Garry PJ. Changes in nutritional status and patterns of morbidity among free-living elderly persons: a 10-year longitudinal study. Nutrition 1997; 13 (6): 515-9.

25. Lasheras C, González C, García A, Patterson A, Fernández S. Dietary intake and biochemical indicators of nutritional status in an elderly institutionalized and noninstitutionalized population. Nutrition Research 1999; 19 (9): 1299-312.

26. Coelho Filho JM, Ramos LR. Epidemiologia do envelhecimento no Nordeste do Brasil: resultados de inquérito domiciliar. Rev Saúde Pública 1999; 33 (5): 44553.

27. Telarolli Jr R; Machado JCMS; Carvalho F. Perfil demográfico e condições sanitárias dos idosos em área urbana do Sudeste do Brasil. Rev Saúde Pública 1996; 30 (5): 485-98. 
28. Organização Pan-Americana da Saúde. Envelhecimento: Mitos na berlinda. Programa Envelhecimento e Saúde. Escritório Regional da Organização Mundial da Saúde. 1999.

29. Schlenker ED. Who are the aging? In: Nutrition in Aging. $3^{\text {rd }}$ ed. McGraw-Hill: Boston; 1998. p. 1-16.

30. WHO. World Health Organization. Obesity: preventing and managing the global epidemic - report of a WHO consultation on obesity. Geneva: WHO; 3-5 June 1997.

31. Marucci MFN. Aspectos nutricionais e hábitos alimentares de idosos matriculados em ambulatório geriátrico [tese de doutorado]. São Paulo: Faculdade de Saúde Pública da USP; 1992.

32. Cervato AM, Mazzilli RN, Martins IS, Marucci MFN. Dieta habitual e fatores de risco para doenças cardiovasculares. Rev Saúde Pública 1997; 31 (3): 227-35.

33. Barreto SM, Passos VMA, Costa MFFL. Obesity and underweight among Brazilian elderly. The Bambuí Health and Aging Study. Cad Saúde Pública 2003; 19 (2): 605-12.

34. Marucci MFN, Barbosa AR. Estado nutricional e capacidade física. In: Lebrão ML e Duarte YAO (Orgs.). $S A B E$ - Saúde, Bem estar e Envelhecimento. O projeto SABE no município de São Paulo: Uma abordagem inicial. Brasília: Organização Pan-Americana da Saúde. 2003. p. 95-117.
35. Groot LCPGM, Sette S, Zajkás G, Carbajal A, Cruz JAA. Nutritional status: anthropometry. Eur J Clin Nutr 1991; 45 (S3): 31-42.

36. Ukoli FA, Bunker CH, Fabio A, Olomu AB, Egbagbe EE, Kuller LHI. Body fat distribution and other anthropometric blood pressure correlates in a Nigerian urban elderly population. Cent J Med 1995; 41(5): 54-161.

37. Velazquez AMDC, Castillo ML, Camacho EI, Zepeda MAS, Robledo LMG, Moysen PC. Estudio antropométrico en un grupo de hombres e mujeres de la tercera edad em la Ciudad de México. Salud Publ Mex 1996; 38: 466-74.

38. Montilla RNG, Marucci MFN, Aldrighi JM. Avaliação do estado nutricional e do consumo alimentar de mulheres no climatério. RevAssoc Med Bras 2003; 49(1): 91-5.

39. Troiano RP, Frongillo Jr EA, Sobal J, Levitsky DA. The relationship between body weight and mortality: a quantitative analysis of combined information from existing studies. Int J Obesity 1996; 20: 63-75.

40. Ramos, LR. Fatores determinantes do envelhecimento saudável em idosos residentes em centro urbano: Projeto Epidoso, São Paulo. Cad Saúde Pública 2003; 19(3): 79398.

Recebido em: 22/08/06 Versão final reapresentada em: 27/02/07 Aprovado em: 19/03/07 